\title{
Recaída bioquímica en cáncer de próstata de bajo riesgo tratados con prostatectomía radical y linfadenectomía pélvica
}

\section{Biochemical Relapse in Low Risk Prostate Cáncer Treated with Radial Prostatectomy and Pelvic Lymphadenectomy} \author{
Juanita González ${ }^{3}$ \\ ${ }^{1}$ Departamento de Urología, IV año, Fundación Universitaria Sanitas, \\ Bogotá, Colombia \\ ${ }^{2}$ Clínica Urología Oncológica, Instituto Nacional de Cancerología, \\ Bogotá, Colombia \\ 3 Faculdad de Medicina, Universidad Nacional de Colombia, Bogotá, \\ Colombia
}

Nataly González ${ }^{1}$ Rodolfo Varela ${ }^{2,3}$ Carlos Céspedes ${ }^{2}$ Byron López de Mesa²,3

Urol Colomb 2020;29:123-128.

\author{
Address for correspondence Nataly González González, MD, \\ Departmento de Urología IV año, Fundación Universitaria Sanitas, \\ Bogotá, Colombia (e-mail: natalygonzalez_1@hotmail.com; \\ natalygonzalez1@gmail.com).
}

\section{Resumen \\ Palabras Clave \\ - cáncer de próstata \\ - riesgo \\ - bioquímico \\ - prostatectomía \\ - linfadenectomía \\ - recaída \\ - márgenes quirúrgicos positivos}

Objetivo La linfadenectomía pélvica ampliada es el método más confiable para identificar compromiso ganglionar en cáncer de próstata, sin embargo, la morbilidad, el tiempo quirúrgico, el papel terapéutico y las complicaciones, han sido temas de debate. El objetivo del estudio fue describir las características clínicas y patológicas de acuerdo con la presentación de recaída bioquimíca de los pacientes con cáncer de próstata de bajo riesgo tratados con prostatectomía radical más linfadenectomía pélvica ampliada.

Métodos Estudio descriptivo longitudinal retrospectivo en una cohorte de pacientes con cáncer de próstata de bajo riesgo tratados quirúrgicamente, entre enero 2006 hasta diciembre 2016. Se revisaron 210 historias clínicas, 178 cumplían los criterios de inclusión: PSA $<10 \mathrm{ng} / \mathrm{mL}$; Gleason $<6$, cT1-cT2a, revisión de las biopsias de próstata y procedimientos quirúrgicos realizados en la misma institución. Las variables a evaluar: porcentaje de compromiso tumoral, invasión linfovascular, concordancia de gleason, numero de ganglios resecados, ganglios positivos, densidad ganglionar, recaída bioquímica. received

February 16, 2018

accepted

September 29, 2018
DOI https://doi.org/

10.1055/s-0038-1675425.

ISSN 0120-789X.

eISSN 2027-0119.
Copyright ( 2020, Sociedad Colombiana License terms de Urología. Publicado por Thieme Revinter Publicações Ltda., Rio de Janeiro, Brazil. Todos los derechos reservados.

() (1) $\Theta \circledast$ 
Resultados 178 pacientes con: 64 años, $62 \% \mathrm{~T} 1 \mathrm{c}$, psa de 6,37, compromiso de biopsia 23\%. El $47 \%$ estaban subestadificados por gleason, con un promedio de ganglios resecados de 21 , el compromiso ganglionar se encontró en un $3 \%$, los bordes positivos en un $34 \%$ y la recaída bioquímica en un $19 \%$. De los 33 pacientes con recaída bioquímica, el $6 \%$ tenían ganglios positivos y el $79 \%$ tenían bordes positivos, comparado con los que no recayeron: el $1 \%$ tenían ganglios positivos y el $23 \%$ eran $\mathrm{R} 1$, mientras que la invasión linfovascular solo estuvo presente en el $6 \%$ vs el $1 \%$ sin recaída. El tiempo a la recaída fue de 26 meses.

Conclusiones El compromiso ganglionar en cáncer de próstata es bajo, pero la subestadificación es alta. En el grupo de recaída bioquímica se observó que la mayoría de los pacientes presentaban bordes positivos y una subestadificacion del Gleason.

\section{Abstract}

\section{Keywords}

- prostate cancer

- risk

- biochemical

- prostatectomy

- lymphadenectomy

- relapse

- positive surgical margins
Objective Extended pelvic lymphadenectomy is the most reliable method to identify lymph node involvement in prostate cancer, however, the morbidity, surgical time, therapeutic role and complications have been debated. The objective of this study was to describe the clinical and pathological characteristics according to the presentation of biochemical relapse in patients with low-risk prostate cancer treated with radical prostatectomy and extended pelvic lymphadenectomy.

Methods A retrospective longitudinal descriptive study in a cohort of patients with low-risk prostate cancer treated surgically, from January 2006 to December 2016. 210 clinical records were reviewed, 178 met the inclusion criteria: PSA $<10 \mathrm{ng} / \mathrm{mL}$; Gleason $<6$, cT1 - cT2a, review of prostate biopsies and surgical procedures performed in the same institution. The variables to be evaluated: percentage of tumor commitment, lymphovascular invasion, gleason score, number of resected nodes, positive lymph nodes, lymph node density, biochemical relapse.

Results 178 patients with: 64 years, $62 \% \mathrm{~T} 1 \mathrm{c}$, psa of 6.37 , commitment of biopsy $23 \%$. $47 \%$ were understaged by gleason, with an average of 21 resected nodes, nodal involvement was found in $3 \%$, positive edges in $34 \%$ and biochemical relapse in $19 \%$. Of the 33 patients with biochemical relapse, $6 \%$ had positive lymph nodes and $79 \%$ had positive margins, compared with those who did not relapse: $1 \%$ had positive lymph nodes and $23 \%$ were $\mathrm{R} 1$, whereas lymphovascular invasion was only present in $6 \% \mathrm{vs} 1 \%$ without relapse. The time to relapse was 26 months.

Conclusions The lymph node involvement in prostate cancer is low, but sub-staging is high. Patients with biochemical relapse they had positive borders and a sub-staging of the Gleason.

\section{Introducción}

El riesgo de ganglios positivos en manejo quirúrgico del cáncer de próstata varía entre un $3 \%$ a un $24 \%$ y la linfadenectomía pélvica ampliada (ePLND) es el método más confiable para determinar la afectación ganglionar en los pacientes con cáncer de próstata, debido a que según el compromiso linfático, se pueden cambiar conductas en el tratamiento. Por otra parte, algunos estudios han mostrado que ese procedimiento, tiene beneficios no solo diagnósticos, sino también, terapéuticos. Sin embargo, la morbilidad, el tiempo quirúrgico requerido y las complicaciones, han sido tema de debate en los últimos 15 años. En una revisión sistemática de la literatura por Harbin, en el 2014, se documentó una tasa que varía entre el 4,1\% y el 19,8\% para complicaciones secundarias a ePLND. ${ }^{1}$

La disección de los ganglios linfáticos está recomendada en pacientes con cáncer de próstata confinado, con psa $>10 \mathrm{ng} / \mathrm{ml}$, gleason $>7$ estadios $>$ T2a o basado en los resultados de los nomogramas, de acuerdo a cada caso. Por otra parte, desde el 2013, las guías europeas de urología han recomendado realizar ese procedimiento quirúrgico, para hombres con cáncer de próstata riesgo intermedio y alto. Inicialmente, en pacientes con cáncer de próstata de bajo riesgo, la ePLND no se realiza de rutina, dado el bajo compromiso de metástasis ganglionares. ${ }^{2,3}$

En el artículo original de D̀Amico en 1998, se estratificaron los riesgos de recaída bioquímica en cáncer de próstata órgano 
confinado de acuerdo al PSA pretratamiento, al Gleason bioptico y al estadio cT, eso se llevó a cabo en pacientes que fueron tratados con prostatectomía radical, radioterapia o terapia de implante con o sin neoadyuvancia. Se estudiaron 1872 pacientes, de ellos, 888 fueron manejados con prostatectomia radical, 218 con radiación intersticial y 766 con radioterapia, el grupo de bajo riesgo se catalogó como estadio T1c, T2a, PSA menor a $10 \mathrm{ng} / \mathrm{ml}$ y Gleason score $<6$, el riesgo relativo (RR) de falla del PSA en bajo riesgo fue de 1,1 (0.5-2.7) para radioterapia externa, de $1,1(0,3-3,6)$ para radiación intersticial o implante y de 0,5 (0.1-1.9) para implante más neoadyuvancia comparado con los pacientes tratados con prostatectomía radical. En el seguimiento se demostró que los pacientes de bajo riesgo podían tener aumentos de PSA tardíos, generalmente cursan con progresión lenta del PSA, en los casos que ocurre, alrededor de los 5 años pos tratamiento. ${ }^{4}$

Objetivo: describir las características clínicas y patológicas de acuerdo con la presentación de recaída bioquímica de los pacientes con cáncer de próstata de bajo riesgo tratados con prostatectomía radical más linfadenectomía pélvica ampliada.

\section{Métodos}

Estudio descriptivo longitudinal retrospectivo en una cohorte de pacientes con cáncer de próstata de bajo riesgo. Las fuentes principales de información fueron el registro de procedimientos quirúrgicos y las historias clínicas. Inicialmente se revisó la base de datos de todos los procedimientos quirúrgicos del instituto nacional de cancerología, se seleccionaron los pacientes sometidos a prostatectomía radical más linfadenectomía pélvica desde enero 2006 hasta diciembre 2016. El seguimiento realizado dependió del curso de la enfermedad de cada paciente, si se encontraba estable, el seguimiento se realizó con la anamnesis, examen físico y exámenes de laboratorio cada 3 meses por los primeros cinco años de diagnóstico del cáncer de próstata y posteriormente cada 6 meses por los siguientes cinco años, el seguimiento más largo encontrado fue a 10 años. La ePLND incluyó los ganglios iliacos externos, hipogástricos, iliobturadores de forma bilateral teniendo como límites el nervio ilioinguinal lateralmente, la pared vesical medialmente, el ligamento de Cooper a nivel distal y la bifurcación de la arteria iliaca común a nivel proximal. ${ }^{5,6}$ Por protocolo, el tejido obtenido en la disección fue enviado para estudio patológico de manera separada según los grupos ganglionares.

Se encontraron 210 historias clínicas revisadas de pacientes con diagnóstico inicial pre quirúrgico de cáncer de próstata de bajo riesgo. El método de selección fue no probabilístico y se incluyó la totalidad de pacientes que cumplían los criterios de selección. Se aplicaron los criterios de inclusión: pacientes clasificados como riesgo bajo de acuerdo con la clasificación de riesgo de D'amico: PSA < $10 \mathrm{ng} / \mathrm{mL}$; Gleason $<6$, cT1-cT2a, revisión de las biopsias de próstata extrainstitucionales por el servicio de patología del instituto nacional de cancerología, 210 pacientes cumplían con los criterios de inclusión. Se excluyeron 5 casos de la revisión de patología de la biopsia después de la prostatectomía radical más linfadenectomía

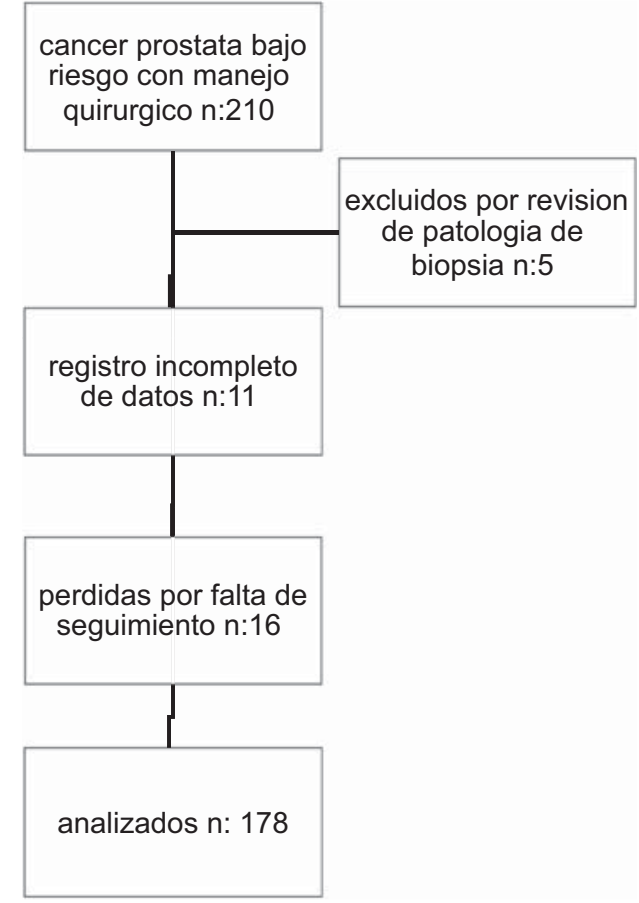

Fig. 1 Flujograma de pacientes incluidos en el estudio.

pélvica y 11 pacientes tenían registro incompleto de datos, en cuanto a las perdidas por falta de seguimiento fueron en 16 casos, en total se excluyeron 32 pacientes y los casos analizados fueron 178 pacientes (-Fig. $\mathbf{1}$ ).

La variable dependiente del estudio fue recaída bioquímica y se tuvieron en cuenta variables de exposición como el porcentaje de compromiso tumoral, la invasión linfovascular, la concordancia del gleason en la biopsia con el producto de la prostatectomía, el número de ganglios resecados, los ganglios positivos y la densidad ganglionar. No se contemplan posibles variables de confusión debido al diseño del estudio y al análisis descriptivo. Debido a que es un estudio retrospectivo, las variables se tomaron de la historia clínica y bases de datos de laboratorio o patología, de acuerdo al protocolo de la institución de III nivel. Para el análisis se usaron medidas de frecuencia, tendencia central y dispersión según tipo de variable. La distribución normal se evalúo mediante la prueba de Kolmogorov-Smirnov. Se usaron las pruebas no paramétricas chi cuadrado o Fischer y t de student o Mann-Whitney (según distribución de variables cuantitativas) para realizar las comparaciones entre los grupos (pacientes con y sin recaída bioquímica). Se registraron las variables en una base de datos en Excel y el análisis estadístico de los datos mediante el programa Stata 10.0 .

La recaída bioquímica se consideró con dos niveles consecutivos de PSA igual o superiores a $0,2 \mathrm{ng} / \mathrm{mL}$; la densidad ganglionar se calculó mediante el número de ganglios positivos sobre el número de ganglios resecados. Por otra parte, el tiempo de seguimiento se extrajo desde la fecha de la cirugía hasta la fecha de la última consulta, mientras que el tiempo hasta la recaída, se obtuvo desde la 
fecha de la prostatectomía, hasta la fecha del primer valor de PSA igual o superior a $0.2 \mathrm{ng} / \mathrm{mL}^{7-9}$

\section{Resultados}

Los pacientes con cáncer de próstata de bajo riesgo llevados a prostatectomía radical más linfadenectomía pélvica ampliada, tenían como características de base una edad promedio de 64 años (44-78), el 62\% eran cT1c, el PSA promedio era de $6,37 \mathrm{ng} / \mathrm{ml}(1,32-10)$, el porcentaje de compromiso del tumor en la biopsia fue del $23 \%$ y en promedio se resecaron 21 ganglios linfáticos (8-51). El porcentaje de subestadificación del Gleason en pacientes con cáncer de próstata de riesgo bajo fue de $35 \% 3+4$ (grado- grupo 2), $7 \% 4+3$ (grado- grupo 3), $5 \%$ presentaba patrón terciario. En total, la subestadificación para el Gleason fue del 47\%. (- Tabla 1 ).

Tabla 1 Características de base: cáncer de próstata de bajo riesgo tratados con prostatectomía radical y linfadenectomía pélvica

\begin{tabular}{|c|c|}
\hline Total de pacientes (n) & 178 \\
\hline Edad (mediana-RIQ) & $64(44-78)$ \\
\hline \multicolumn{2}{|l|}{ Estadio TNM inicial } \\
\hline cT1c & $111(62 \%)$ \\
\hline cT2a & $67(38 \%)$ \\
\hline PSA inicial (ng/mL) & $6,37(1,32-10)$ \\
\hline Biopsia \% compromiso & $23 \%(2-80 \%)$ \\
\hline Ganglios resecados & $21(8-51)$ \\
\hline \multicolumn{2}{|l|}{ Gleason (\%) } \\
\hline $3+3$ (grado- grupo 1$)$ & $95(53 \%)$ \\
\hline $3+4$ (grado- grupo 2 ) & $62(35 \%)$ \\
\hline $4+3$ (grado- grupo 3 ) & $13(7 \%)$ \\
\hline $3+3+4$ & $5(3 \%)$ \\
\hline $4+3+5$ & $3(2 \%)$ \\
\hline \multicolumn{2}{|l|}{ Estadio TNM patológico } \\
\hline $\mathrm{pT} 2 \mathrm{a}$ & $23(13 \%)$ \\
\hline $\mathrm{pT2b}$ & $6(3 \%)$ \\
\hline pT2c & $106(60 \%)$ \\
\hline pT3a & $38(21 \%)$ \\
\hline pT3b & $5(3 \%)$ \\
\hline \multicolumn{2}{|l|}{ Compromiso ganglionar } \\
\hline NO & $173(97 \%)$ \\
\hline N1 & $5(3 \%)$ \\
\hline \multicolumn{2}{|l|}{ Bordes positivos } \\
\hline R0 & $118(66 \%)$ \\
\hline R1 & $60(34 \%)$ \\
\hline \multicolumn{2}{|l|}{ Recaída bioquímica } \\
\hline Sí & $33(19 \%)$ \\
\hline No & $145(81 \%)$ \\
\hline
\end{tabular}

En cuanto a las características clínicas y patológicas de los 33 pacientes con recaída bioquímica post prostatectomía radical más linfadenectomía pélvica ampliada, que equivale al 19\% de la población total, se encontró, el 58\% eran cT1, el $42 \%$ era cT2a, con un PSA promedio de $6,6 \mathrm{ng} / \mathrm{ml}(3,7-9,7)$, el $70 \%$ no presentaba invasión linfovascular, los ganglios resecados en promedio fueron $22(10-48)$ y el $61 \%$ tenían una patología Gleason $3+4,9 \%$ para Gleason $4+3$ y un total de subestadificación de $70 \%$ comparado con el $43 \%$ de los que no recayeron, el $79 \%$ presentaba bordes positivos frente a un $23 \%$ de los pacientes que no recayeron, el tiempo promedio a la recaída fue de 26,4 meses (-Tabla 2 ).

Por otra parte, solo el 3\% de los pacientes, presentaron ganglios linfáticos positivos, con un promedio de resección de 21 ganglios, en cuanto a las regiones anatómicas con mayor

Tabla 2 Características clínicas y patológicas de pacientes con y sin recaída bioquímica

\begin{tabular}{|c|c|c|}
\hline & $\begin{array}{l}\text { Recaída } \\
\text { bioquímica } \\
n=33(\%)\end{array}$ & $\begin{array}{l}\text { Libre de } \\
\text { recaída } \\
n=145(\%)\end{array}$ \\
\hline Edad Mediana (RIQ) (años) & $66(46-76)$ & $63(44-78)$ \\
\hline \multicolumn{3}{|l|}{ Estadio TNM inicial (\%) } \\
\hline cT1c & $19(58 \%)$ & $92(63 \%)$ \\
\hline cT2a & $14(42 \%)$ & $53(37 \%)$ \\
\hline PSA ng/ml & $6,22(3,7-9,7)$ & $6,28(1,32-10)$ \\
\hline Biopsia \% compromiso & $23 \%$ & $22 \%$ \\
\hline Ganglios promedio (n) & $22(10-48)$ & $20(8-51)$ \\
\hline \multicolumn{3}{|l|}{ Gleason (\%) } \\
\hline $3+3$ (grado- grupo 1$)$ & $10(30 \%)$ & $82(57 \%)$ \\
\hline $3+4$ (grado- grupo 2) & 20 (61\%) & $51(35 \%)$ \\
\hline $4+3$ (grado- grupo 3) & $3(9 \%)$ & $10(7 \%)$ \\
\hline $4+3+5$ & $0(\%)$ & $2(1 \%)$ \\
\hline \multicolumn{3}{|l|}{ Estadio TNM patológico (\%) } \\
\hline pT2a & $6(18 \%)$ & $16(11 \%)$ \\
\hline pT2b & $1(3 \%)$ & $6(4 \%)$ \\
\hline $\mathrm{pT} 2 \mathrm{c}$ & $14(43 \%)$ & $92(64 \%)$ \\
\hline pT3a & $9(27 \%)$ & $29(20 \%)$ \\
\hline pT3b & $3(9 \%)$ & $2(1 \%)$ \\
\hline \multicolumn{3}{|l|}{ Compromiso ganglionar (\%) } \\
\hline No & $31(94 \%)$ & $142(98 \%)$ \\
\hline N1 & $2(6 \%)$ & $3(2 \%)$ \\
\hline \multicolumn{3}{|l|}{ Bordes positivos } \\
\hline Ro & $7(21 \%)$ & $111(77 \%)$ \\
\hline R1 & $26(79 \%)$ & $34(23 \%)$ \\
\hline \multicolumn{3}{|l|}{ Invasión linfovascular } \\
\hline Sí & $2(6 \%)$ & $2(1 \%)$ \\
\hline No & $23(70 \%)$ & $137(95 \%)$ \\
\hline No reporte & 8 (24\%) & $6(4 \%)$ \\
\hline $\begin{array}{l}\text { Tiempo a la recaída } \\
\text { (meses) }\end{array}$ & 26,4 & no aplica \\
\hline Mortalidad & 0 & $2(1,1 \%)$ \\
\hline
\end{tabular}


extracción de ganglios fue la iliobturadora, sin embargo, en nuestro estudio, la mayoría de las metástasis ganglionares se encontraban localizadas en la región hipogástrica e iliaca externa y de los pacientes con recaída bioquímica, el 6\% tenían ganglios positivos, mientras que los casos libres de recaída solo presentaron un $2 \%$ de compromiso ganglionar.

\section{Discusión}

En el presente estudio se observó una tasa de subestadificacion del $47 \%$ de los pacientes con cáncer de próstata de bajo riesgo llevados a prostatectomía radical más linfadenectomía pélvica ampliada, de los cuales 33 pacientes (19\%), presentaron recaída bioquímica de la enfermedad a los 26,4 meses y de ese grupo específicamente la tasa de subestadificacion fue del $70 \%$ y un $79 \%$ de bordes positivos. En cuanto a la linfadenectomía pélvica ampliada, en promedio se extrajeron 21 ganglios, de los cuales solo el $3 \%$ presentaba compromiso tumoral.

La decisión de realizar la linfadenectomía pélvica, depende de varios factores clínicos, patológicos y la predicción del riesgo de metástasis ganglionares basado en diferentes nomogramas. Sin embargo, cabe resaltar que los cálculos más utilizados, se realizaron a partir de grupos de pacientes con linfadenectomía limitada y eso puede generar falsos negativos y subestimar el riesgo. ${ }^{10-12}$

En el caso de los nomogramas de Briganti y Godoy, ellos utilizaron pacientes con linfadectomia ampliada. Recientemente, Gandaglia en el 2017, realizó un nomograma para predecir la invasión tumoral a ganglios linfáticos, de acuerdo a un modelo multivariable incluyendo psa, estadio clínico, gleason biopsia en grado grupo, porcentaje de cores con alto grado y cores con bajo grado, con una predicción calculada de $90 \%$ que se realiza en caso de riesgo calculado mayor al 7\%. ${ }^{13-17}$

Un estudio colombiano multicéntrico retrospectivo en el 2016, sobre el cáncer de próstata en todos los riesgos a quienes se les realizó prostatectomía radical más linfadenectomía pelvica ampliada, con 2876 ganglios, en promedio de 20,9 ganglios por paciente, se encontraron un compromiso ganglionar del $10,22 \%$, pero no encontraron ganglios positivos en el grupo de bajo riesgo. De los pacientes con $>2 \%$ de riesgo basado en nomogramas, solo el 19\% presentó compromiso ganglionar. ${ }^{18}$ Comparado con nuestro estudio, de los pacientes con compromiso ganglionar y con recaída bioquímica, se encontró que el 100\% tenían bordes positivos, confrontado con el 33\% de los ganglios positivos, sin recaída.

Si le aplicamos los nomogramas de Partin y de Briganti, a los cinco pacientes que presentaron ganglios positivos, solo dos personas tenían riesgo de compromiso ganglionar, uno de $4 \%$ y otro de $19 \%$.

Por otra parte, en un estudio de Heidenreinch en el 2002, con 203 pacientes divididos en dos grupos de acuerdo a la linfadenectomía estándar vs extendida, en prostatectomía radical retropubica. Los hallazgos histopatológicos se compararon con el psa, el estadio y la biopsia previa. Se tuvieron en cuenta, las complicaciones, el número de ganglios linfáticos resecados y el tiempo operatorio. Se reportó una tasa de compromiso ganglionar de un 26,2\% en linfadenectomía pelvica extendida, con un promedio resecado de 28 ganglios linfáticos, se encontraron ganglios positivos en la región iliaca interno y presacros a pesar de que los obturadores fueron negativos. En la presente publicación, se encontró una tasa de compromiso ganglionar del 3\%, con un promedio de 22 ganglios resecados y un porcentaje de subestadificación del $47 \%{ }^{19}$

En un estudio por Imnadze en el 2016 sobre las características patológicas adversas y los resultados oncológicos con 2660 pacientes de todos los riesgos según D̀amico, específicamente con 1102 de bajo riesgo, se observó que los Gleason patológicos $4+3$ tenían un riesgo de recurrencia de la enfermedad a 5 años del $13 \% .{ }^{20}$ Mientras Ji, en el 2012 desarrolló un estudio con 360 pacientes con cáncer de próstata localizado de todos los riesgos que recibieron tratamiento quirúrgico con linfadenectomía estándar o ampliada, la supervivencia libre de progresión bioquímica para el grupo de bajo riesgo con linfadenectomía limitada fue del 90,1\%, mientras que la ampliada fue del $91,3 \%$. Comparado con nuestro estudio, el porcentaje de recaída bioquímica fue del $19 \%$ y de ellos, el $70 \%$ estaban subestadificados, lo cual plantea la hipótesis del efecto curativo de la linfadenectomía pélvica.

En un artículo de Watkins en el 2014, para determinar si características patológicas adicionales generan riesgo en pacientes con márgenes quirúrgicos positivos (R1) en prostatectomía radical, se realizó con 279 pacientes con un seguimiento de 53 meses y se mostró que un $27 \%$ de los pacientes $\mathrm{R} 1$ presentaban recaída bioquímica contra un $4 \%$ para RO, con un HR 4,9 IC 95\% 2,4-10 y una $p<0,001$. También describen asociaciones con la invasión linfovascular y la recaída del PSA. En nuestro estudio, el $79 \%$ de los pacientes que recayeron eran $\mathrm{R} 1$, mientras que la invasión linfovascular solo estuvo presente en un $6 \%{ }^{21}$

La principal limitación de nuestro estudio, se centra en la diferencia en el tiempo de seguimiento de los pacientes, debido a que al corresponder a un estudio retrospectivo la información disponible sobre el seguimiento de los pacientes es limitada y todos los pacientes no tienen el mismo tiempo de seguimiento para evaluar el desenlace, por esta razón, el estudio describe las diferencias encontradas al comparar los dos grupos (presencia o no de recaída bioquímica), que se aplican a la población de estudio y deben ser evaluadas como posibles factores asociados mediante futuros estudios analíticos y prospectivos. En relación a la calidad de los datos, se considera que las fuentes de información usadas (laboratorio y patología), son de alta calidad debido a procedimientos estandarizados usados en la práctica diaria.

\section{Conclusiones}

La linfadenectomía pélvica es el método más fiable para estadificar la enfermedad en cuanto a compromiso ganglionar. La región con mayor extracción de ganglios fue la iliobturadora, no obstante, la localización de metástasis ganglionares en nuestro estudio fue hipogástrica e iliaca externa, lo que recomendaría el uso de la linfadenectomía 
pélvica ampliada. El 70\% de las personas que recayeron estaban subestadificados, lo cual plantea la hipótesis de las limitaciones de los nomogramas y del efecto terapéutico de la linfadenectomía pélvica, por otra parte, se encontró relación entre la recaída bioquímica y los bordes positivos. Se debe plantear para un futuro, estudios futuros estudios analíticos y prospectivos, con mayor poder estadístico que incluyan complicaciones y tiempo operatorio.

\section{Responsabilidades Éticas}

Protección de personas y animales. Los autores declaran que para esta investigación no se han realizado experimentos en seres humanos ni en animales.

Confidencialidad de los datos. Los autores declaran que han seguido los protocolos de su centro de trabajo sobre la publicación de datos de pacientes.

Derecho a la privacidad y consentimiento informado. Los autores declaran que en este artículo no aparecen datos de pacientes.

\section{Referencias}

1 Harbin AC, Eun DD. The role of extended pelvic lymphadenectomy with radical prostatectomy for high-risk prostate cancer. Urol Oncol 2015;33(05):208-216

2 European Association of Urology 2017 Guidelines on Prostate Cancer. Available at: uroweb.org.

3 National Comprehensive Cancer Network Guidelines Version 1.2017 Prostate Cancer. Available at: nccn.org.

4 D'Amico AV, Whittington R, Malkowicz SB, et al. Biochemical outcome after radical prostatectomy, external beam radiation therapy, or interstitial radiation therapy for clinically localized prostate cancer. JAMA 1998;280(11):969-974

5 Allaf ME, Palapattu GS, Trock BJ, Carter HB, Walsh PC. Anatomical extent of lymph node dissection: impact on men with clinically localized prostate cancer. J Urol 2004;172(5 Pt 1):1840-1844

6 Heidenreich A, Ohlmann CH, Polyakov S. Anatomical extent of pelvic lymphadenectomy in patients undergoing radical prostatectomy. Eur Urol 2007;52(01):29-37

7 Barba J, Brugarolas X, Tolosa E, et al. Factores influyentes en el tiempo hasta la progresión bioquímica después de prostatectomía radical. Actas Urol Esp 2011;35(04):201-207

8 Milonas D, Kinčius M, Skulčius G, Matjošaitis AJ, GudinavičienĖ I, Jievaltas M. Evaluation of D'Amico criteria for low-risk prostate cancer. Scand J Urol 2014;48(04):344-349

9 Petrelli F, Vavassori I, Cabiddu M, et al. Predictive Factors for Reclassification and Relapse in Prostate Cancer Eligible for Active
Surveillance: A Systematic Review and Meta-analysis. Urology 2016;91:136-142

10 Partin AW, Kattan MW, Subong EN, et al. Combination of prostatespecific antigen, clinical stage, and Gleason score to predict pathological stage of localized prostate cancer. A multiinstitutional update. JAMA 1997;277(18):1445-1451

11 Abdollah F, Sun M, Suardi N, et al; National Comprehensive Cancer Network. National Comprehensive Cancer Network practice guidelines 2011: Need for more accurate recommendations for pelvic lymph node dissection in prostate cancer. J Urol 2012;188 (02):423-428

12 Andrade CA, Hernández CE, Sejnaui JE, Caicedo J. Linfadenectomía pélvica ilio-obturadora por laparoscopia en el cáncer de próstata. Urol Colomb. 2010;XIX:55-63

13 Lendínez-Cano G, Alonso-Flores J, Beltrán-Aguilar V, Cayuela A, Salazar-Otero S, Bachiller-Burgos J. Comparación de datos anatomopatológicos entre biopsia de próstata y pieza de prostatectomía radical en pacientes con cáncer de bajo y muy bajo riesgo. Actas Urol Esp 2015;39(08):482-487

14 Briganti A, Larcher A, Abdollah F, et al. Updated nomogram predicting lymph node invasion in patients with prostate cancer undergoing extended pelvic lymph node dissection: the essential importance of percentage of positive cores. Eur Urol 2012;61(03):480-487

15 Godoy G, Chong KT, Cronin A, et al. Extent of pelvic lymph node dissection and the impact of standard template dissection on nomogram prediction of lymph node involvement. Eur Urol 2011; 60(02):195-201

16 Gandaglia G, Fossati N, Zaffuto E, Bandini M, Dell'Oglio P, Bravi CA et al. Development and Internal Validation of a Novel Model to Identify the Candidates for Extended Pelvic Lymph Node Dissection in Prostate Cancer. Eur Urol 2017:0302-2838

17 Beauval JB, Cabarrou B, Gandaglia G, et al. External validation of a nomogram for identification of pathologically favorable disease in intermediate risk prostate cancer patients. Prostate 2017;77 (08):928-933

18 Ramos JG, Caicedo JI, Cataño JG, et al. Linfadenectomía pélvica extendida en pacientes con cáncer de próstata clínicamente localizado: estudio observacional prospectivo. Actas Urol Esp 2016;40(07):446-452

19 Heidenreich A, Varga Z, Von Knobloch R. Extended pelvic lymphadenectomy in patients undergoing radical prostatectomy: high incidence of lymph node metastasis. J Urol 2002;167(04): $1681-1686$

20 Imnadze M, Sjoberg DD, Vickers AJ. Adverse Pathologic Features at Radical Prostatectomy: Effect of Preoperative Risk on Oncologic Outcomes. Eur Urol 2016;69(01):143-148

21 Watkins JM, Laszewski M, Watkins PL, Dufan TA, Adducci C. Margin involvement at prostatectomy for clinically localized prostate cancer: does a low-risk group exist? Pract Radiat Oncol 2015;5(01):e31-e36 